\title{
\begin{tabular}{l|l} 
Mibraries & DSpace@MIT
\end{tabular}
}

\author{
MIT Open Access Articles
}

\section{SEGUE 1-A COMPRESSED STAR FORMATION HISTORY BEFORE REIONIZATION}

The MIT Faculty has made this article openly available. Please share how this access benefits you. Your story matters.

Citation: Webster, David, Anna Frebel, and Joss Bland-Hawthorn. "SEGUE 1-A COMPRESSED STAR FORMATION HISTORY BEFORE REIONIZATION." The Astrophysical Journal 818, no. 1 (February 9, 2016): 80. (C) 2016 The American Astronomical Society

As Published: http://dx.doi.org/10.3847/0004-637x/818/1/80

Publisher: IOP Publishing

Persistent URL: http://hdl.handle.net/1721.1/102673

Version: Final published version: final published article, as it appeared in a journal, conference proceedings, or other formally published context

Terms of Use: Article is made available in accordance with the publisher's policy and may be subject to US copyright law. Please refer to the publisher's site for terms of use. 


\title{
SEGUE 1-A COMPRESSED STAR FORMATION HISTORY BEFORE REIONIZATION
}

\author{
David Webster $^{1}$, Anna Frebel ${ }^{2}$, and Joss Bland-Hawthorn ${ }^{1}$ \\ ${ }^{1}$ Sydney Institute for Astronomy, School of Physics, University of Sydney, Sydney, NSW 2006, Australia; d.webster@physics.usyd.edu.au \\ ${ }^{2}$ Department of Physics and Kavli Institute for Astrophysics and Space Research, Massachusetts Institute of Technology, Cambridge, MA 02139, USA \\ Received 2015 September 1; accepted 2015 December 23; published 2016 February 9
}

\begin{abstract}
Segue 1 is the current best candidate for a "first galaxy," a system that experienced only a single, short burst of star formation and has since remained unchanged. Here we present possible star formation scenarios that can explain Segue 1's unique metallicity distribution. While the majority of stars in all other ultra-faint dwarfs are within 0.5 dex of the mean $[\mathrm{Fe} / \mathrm{H}]$ for the galaxy, five of the seven stars in Segue 1 have a spread of $\Delta[\mathrm{Fe} / \mathrm{H}]>0.8$ dex. We show that this distribution of metallicities cannot be explained by a gradual buildup of stars, but instead requires clustered star formation. Chemical tagging allows the separate unresolved delta functions in abundance space to be associated with discrete events in space and time. This provides an opportunity to put the enrichment events into a time sequence and unravel the history of the system. We investigate two possible scenarios for the star formation history of Segue 1 using Fyris Alpha simulations of gas in a $10^{7} M_{\odot}$ dark matter halo. The lack of stars with intermediate metallicities $-3<[\mathrm{Fe} / \mathrm{H}]<-2$ can be explained either by a pause in star formation caused by supernova feedback or by the spread of metallicities resulting from one or two supernovae in a low-mass dark matter halo. Either possibility can reproduce the metallicity distribution function (MDF) as well as the other observed elemental abundances. The unusual MDF and the low luminosity of Segue 1 can be explained by it being a first galaxy that originated with $M_{\text {vir }} \sim 10^{7} M_{\odot}$ at $z \sim 10$.
\end{abstract}

Key words: dark ages, reionization, first stars - early universe - galaxies: abundances - galaxies: dwarf - galaxies: formation - stars: abundances

\section{INTRODUCTION}

The formation and evolution of the first stars and galaxies can be studied through observations of nearby dwarf galaxies. Stars preserve their chemical signatures at the time of their formation, such that galaxies with an early truncation of their star formation history can be used to investigate the conditions of the high-redshift universe. These systems are called "first galaxies" or "fossil galaxies" (Ricotti \& Gnedin 2005; Bovill \& Ricotti 2009; Bromm \& Yoshida 2011; Frebel \& Bromm 2012; Frebel et al. 2014; Bland-Hawthorn et al. 2015), reflecting the idea that their stellar population has been frozen in time for $>10$ Gyr. The best candidates for such systems are the ultrafaint dwarfs (UFDs, $L<10^{5} L_{\odot}$ ) discovered in the past decade. Most UFDs contain only old metal-poor stars and appear to have simple star formation histories (Brown et al. 2012, 2014), making them excellent candidates for probing the chemical signatures of the first generations of stars.

There is not yet a consensus on the definition of a "first galaxy," but most authors exclude the $\sim 10^{6} M_{\odot}$ minihalos in which the first Population III stars formed and require a first galaxy to produce and retain a long-lived stellar system (Bromm \& Yoshida 2011; Bland-Hawthorn et al. 2015). Frebel \& Bromm (2012) defined a first galaxy as one which had only a single short burst of star formation. Such a system would be enriched only by Population III stars and would therefore lack the signatures of Type Ia and AGB star enrichment events, resulting in enhanced alpha element abundances and low or zero neutron-capture element abundances.

The best candidate to date for a first galaxy is Segue 1 (Frebel et al. 2014). Segue 1 was discovered by Belokurov et al. (2007) and confirmed as a galaxy by Geha et al. (2009) who showed that it was dark matter-dominated, as well as by Norris et al. (2010b) who showed that it had a large spread in metallicity. The mean metallicity of Segue $1,[\mathrm{Fe} / \mathrm{H}] \approx-2.7$ made it the most metal-poor known galaxy, although the recently discovered Reticulum 2 has a similar mean metallicity (Koposov et al. 2015b; Simon et al. 2015). High-resolution spectroscopy (Frebel et al. 2014) showed that the seven brightest red giants in Segue 1 with metallicities ranging from $[\mathrm{Fe} / \mathrm{H}]=-3.8$ to -1.4 showed $[\alpha / \mathrm{Fe}] \approx 0.5$ and suppressed $[\mathrm{Sr} / \mathrm{H}]$ and $[\mathrm{Ba} / \mathrm{H}]$, suggesting a maximum of one r-process or weak s-process event. This implies that no stars formed from gas enriched by AGB stars, suggesting that star formation in Segue 1 lasted less than the lifetime of 7-8 $M_{\odot}$ stars, $\sim 30-50$ Myr.

The metallicity distribution function (MDF) of Segue 1 is also unusual. While all other known UFDs have metallicity distributions that peak near the mean metallicity and have only a tail of metal-poor or metal-rich stars, Segue 1 shows three stars with $[\mathrm{Fe} / \mathrm{H}] \approx-3.6$, two with $[\mathrm{Fe} / \mathrm{H}] \approx-2.4$, and two with $[\mathrm{Fe} / \mathrm{H}] \approx-1.5$. Five of the seven stars are more metalpoor or metal-rich than $90 \%$ of observed stars in the six UFDs observed by Brown et al. (2014). In this work, we seek to explain the unusual MDF of Segue 1 by exploring possible scenarios for its star formation history. This can give us an insight into the conditions of star formation in the early universe as well as suggest new avenues for determining which systems are likely to be first galaxies.

In the local universe star formation is observed only in clusters (Lada \& Lada 2003). There is some evidence to suggest that this should also be true for the high-redshift universe (Clark et al. 2008; Karlsson et al. 2012, 2013; Larsen et al. 2012). Clusters have homogeneous abundances as long as they are not self-enriching, meaning that the timescale for its star formation must be less than the time of the first supernova. This is supported by observations of clusters, including open clusters (De Silva et al. 2006, 2007; Feng \& Krumholz 2014). 


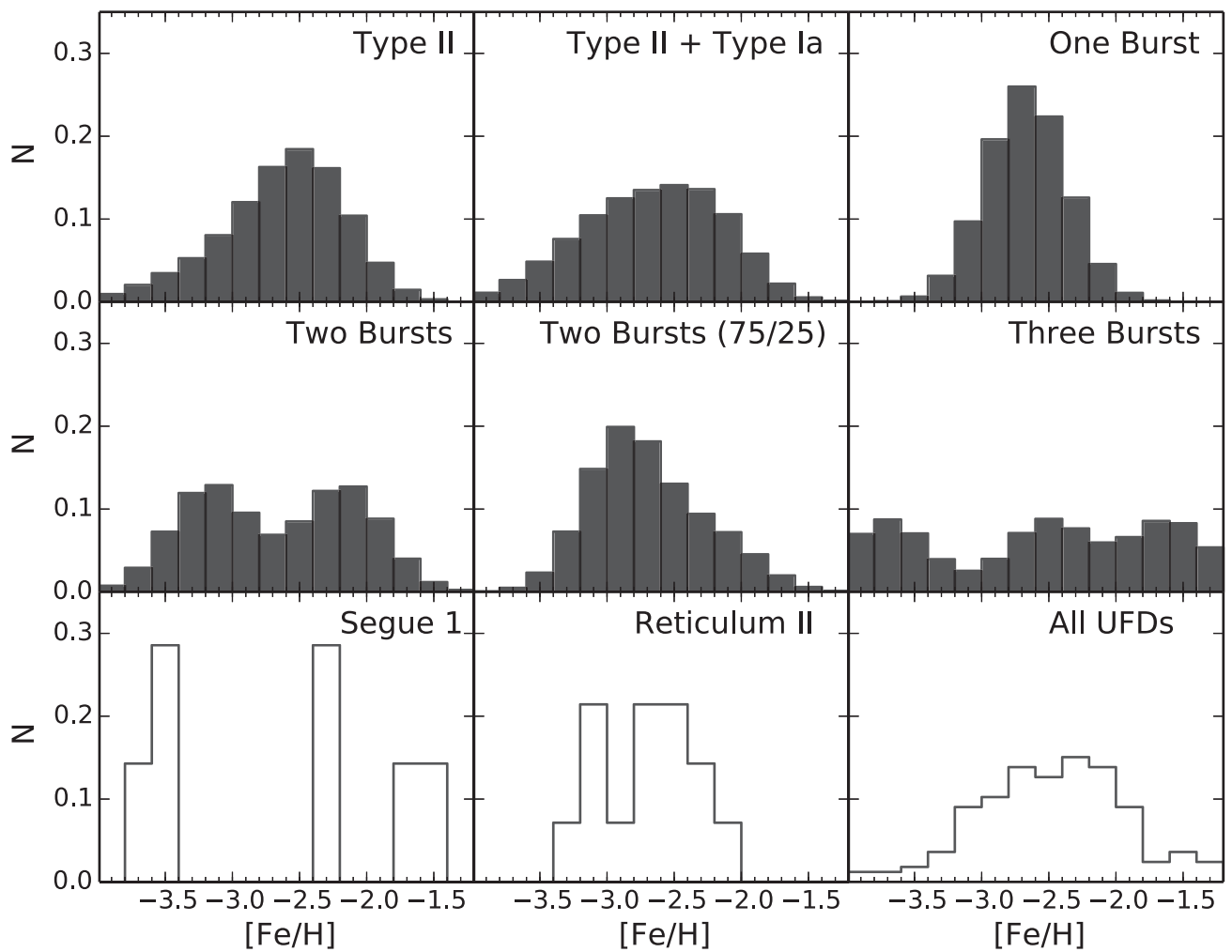

Figure 1. Theoretical normalized MDFs (filled histograms) resulting from a range of possible simple star formation histories along with the normalized MDFs observed in UFDs (unfilled histograms). In the top six panels, the mean $[\mathrm{Fe} / \mathrm{H}] \approx-2.7$, as in Segue 1 and Reticulum 2. The lower three panels show the observed MDFs of individual UFDs as well as the entire observed UFD population (Kirby et al. 2013; Brown et al. 2014; Frebel et al. 2014; Simon et al. 2015).

The chemical homogeneity of clusters has led to the idea of chemical tagging (Bland-Hawthorn et al. 2010b), the search for stars with similar abundances in a range of elements suggesting that they originated from the same cluster. Separate unresolved delta functions in chemical abundance space are associated with events that are discrete in space and time. This provides an opportunity to put these events into a time sequence and determine the detailed enrichment history of a system.

Bland-Hawthorn et al. (2010a) tailored chemical tagging to dwarf galaxies and it was applied by Karlsson et al. (2012) to Sextans, the best candidate for a cluster signature in a dwarf galaxy. They found that three stars with $[\mathrm{Fe} / \mathrm{H}]=-2.7$ contained remarkably similar $\mathrm{Mg}, \mathrm{Ti}, \mathrm{Cr}$, and $\mathrm{Ba}$ abundances. If correct, this group of stars is more metal-poor than any other known star cluster in the Milky Way.

Recently 17 ultra-faint satellites have been discovered in the Southern sky (Drlica-Wagner et al. 2015; Koposov et al. 2015a). It is not yet confirmed whether they are all UFDs, as it is possible that some are globular clusters. The only systems to date with $[\mathrm{Fe} / \mathrm{H}]$ data from spectroscopy are Reticulum 2 and Horologium 1, which are confirmed as UFDs from measurements of the velocity dispersion and the spread in $[\mathrm{Fe} / \mathrm{H}]$ (Koposov et al. 2015b; Simon et al. 2015; Walker et al. 2015). Reticulum 2 is approximately tied with Segue 1 as the most metal-poor galaxy, with $\overline{[\mathrm{Fe} / \mathrm{H}]}=-2.7$. Reticulum 2 is one of the six objects described by Koposov et al. (2015a) as having sizes and luminosities similar to Segue 1. However, as we will see in Section 2, the MDF of Reticulum 2 is more similar to the more luminous UFDs than to that of Segue 1.

In Section 2 we discuss the MDFs produced under various assumptions for the star formation history of a UFD. We compare these with the MDFs of Segue 1, Reticulum 2, and the entire UFD population. In Section 3 we present possible star formation histories for Segue 1, guided by Fyris alpha hydrodynamical simulations of small $\left(M_{\mathrm{vir}}=10^{7} M_{\odot}\right)$ dark matter halos. We discuss our results in Section 4 and summarize in Section 5.

\section{THEORETICAL MDF}

\subsection{Method}

In this section we show MDFs resulting from various star formation histories and compare them with the MDF of Segue 1. Given that Segue 1 is believed to have formed stars for less than $50 \mathrm{Myr}$, it should have a relatively uncomplicated star formation history.

The first two panels of Figure 1 correspond to models of continuous enrichment. In the first panel (labelled "Type II") an equal amount of star formation occurs between each of $14 \mathrm{SNe}$ II. Between time $t$ and $t+\Delta t, 100$ stars form with $[\mathrm{Fe} / \mathrm{H}]$ for a given star $s$ given by:

$$
\begin{aligned}
{[\mathrm{Fe} / \mathrm{H}](s) } & =\log \left(n_{\mathrm{Fe}}(s) / n_{\mathrm{H}}\right)-\log \left(n_{\mathrm{Fe}, \odot} / n_{\mathrm{H}, \odot}\right) \\
& =\log \left(\frac{M_{\mathrm{Fe}_{i}}+y_{\mathrm{Fe}} N t(s)}{\mu_{\mathrm{Fe}} M_{\mathrm{H}}}\right)-4.55+X
\end{aligned}
$$

where $M_{\mathrm{H}}=10^{5} M_{\odot}$ and $M_{\mathrm{Fe}_{i}}$, the initial mass of iron in the gas, is set such that $[\mathrm{Fe} / \mathrm{H}]=-3.5$ at $t=0, \mu_{\mathrm{Fe}}$ is the atomic mass of iron, $N$ is the number of supernova explosions per unit time, $y_{\mathrm{Fe}}=0.08 M_{\odot}$ is the iron yield of each (Type II) supernova, $\log \left(n_{\mathrm{Fe}, \odot} / n_{\mathrm{H}, \odot}\right)=-4.55$ (Asplund et al. 2005), and $X \sim \mathcal{N}\left(0,0.3^{2}\right)$ is a random number selected from a Gaussian distribution with $\mu=0, \sigma=0.3$ representing the 
approximate dispersion in metallicities in the interstellar medium (ISM) (Feng \& Krumholz 2014). $N \Delta t$ is set to equal 1 , such that there is one supernova explosion between $t$ and $t+\Delta t$, the period during which 100 stars form. The "Type II +Ia" model in the second panel is the same, except that for a single value of $t$, taken to be half the total time, $y_{\mathrm{Fe}}=0.8 M_{\odot}$, corresponding to the yield of a SN Ia. The supernova yields are from Iwamoto et al. (1999) and Nomoto et al. (2006). The model was run 100 times and the average taken.

The neutral hydrogen mass $M_{\mathrm{H}}=10^{5} M_{\odot}$ assumed is of the order of the amount of gas in the inner $100 \mathrm{pc}$ of an $M_{\text {vir }}=10^{6.5-7} M_{\odot}$ dark matter halo with a baryon fraction of $10 \%$. As shown in Bland-Hawthorn et al. (2015) such a system can retain the majority of its gas and metals in the face of a single supernova explosion. The gas initial constant metallicity of $[\mathrm{Fe} / \mathrm{H}]=-3.5$, corresponds to the expected metallicity for such a system enriched by a first star (Greif et al. 2007; Frebel $\&$ Bromm 2012). At each timestep the Fe yield of a single SN II is added to the total $\mathrm{Fe}$ of the gas with the neutral hydrogen content assumed to remain constant, such that the metallicity of the gas gradually increases.

The second class of scenarios shown in Panels 3-6 ("One Burst," "Two Bursts," "Two Bursts 75/25" and "Three Bursts") of Figure 1 are simply sums of Gaussian distributions. In all cases the scatter of metallicities within a burst is given by the standard deviation of the Gaussians, set to be 0.3 dex as for the continuous models.

This gives a total of six scenarios:

1. "Type II": continuous enrichment from Type II supernovae as in Equation (1).

2. “Type II + Type Ia": continuous enrichment from SNe II and $\mathrm{SNe}$ Ia as in Equation (1).

3. "One Burst": a single burst of stars with mean $[\mathrm{Fe} / \mathrm{H}]=-2.67$, represented by a single Gaussian.

4. "Two Bursts": the sum of two equally weighted Gaussian distributions with means $[\mathrm{Fe} / \mathrm{H}]=-3.17$ and -2.17 , representing the stars being evenly divided between the bursts.

5. "Two Bursts 75/25": the sum of two Gaussians, one with mean $[\mathrm{Fe} / \mathrm{H}]=-2.87$ and one with -2.17 . The first Gaussian is given a relative weight of three times the second, such that this is a model in which $75 \%$ of the stars form in the lower metallicity burst.

6. "Three Bursts": three evenly weighted Gaussians with mean $[\mathrm{Fe} / \mathrm{H}]=-3.7,-2.5$ and -1.6 , representative of three bursts with the stars evenly divided between the bursts.

The first two scenarios correspond to closed box models with no inflows or outflows where the iron is mixed instantaneously with the neutral hydrogen gas. Star formation is terminated when $\overline{[\mathrm{Fe} / \mathrm{H}]}$ in the stars formed is approximately -2.7 , which is the mean $[\mathrm{Fe} / \mathrm{H}]$ observed in Segue 1 and Reticulum 2. This number of supernovae is consistent with the $1500 M_{\odot}$ of star formation implied for Segue 1 under a Kroupa (2001) IMF (Frebel et al. 2014). Assuming a specific star formation rate of $\sim 10^{-3} M_{\odot} \mathrm{yr}^{-1} \mathrm{kpc}^{-2}$ (Bigiel et al. 2008; Webster et al. 2014) as in the Carina dwarf, this will take 100-200 Myr.

The number of stars in a given $[\mathrm{Fe} / \mathrm{H}]$ bin increases exponentially with increasing $[\mathrm{Fe} / \mathrm{H}]$ until the mean metallicity at which star formation is switched off, because $[\mathrm{Fe} / \mathrm{H}]$ is a logarithmic scale and the $\mathrm{Fe}$ mass is assumed to increase linearly with the hydrogen mass remaining constant. There are a few stars with higher metallicities which result from the assumed dispersion of $[\mathrm{Fe} / \mathrm{H}]$.

While Segue 1 shows no evidence of Type Ia enrichment, we include the second panel ("Type II + Type Ia") to show how SNe Ia alter an MDF. The buildup of metallicities remains exponential for the low-metallicity stars that form first. However, a SN Ia injects a large amount of Fe, resulting in a rapid increase in $\mathrm{Fe}$ that flattens the peak of the $[\mathrm{Fe} / \mathrm{H}]$ distribution.

Scenarios 3-6 represent distinct bursts of star formation, with no self-enrichment within each burst and the gas considered to be well-mixed between each burst. They give an indication of the variety of qualitatively different MDFs that can be constructed using simple bursts. This is motivated by Brown et al. (2014) who used isochrone fitting to determine the star formation history of six UFDs and found that they were best fit by two bursts of star formation, although in three of the galaxies $\geqslant 95 \%$ of the stars were in one of the bursts. Giving the bursts a non-zero duration did not improve the fit. Scenario 3 ("One Burst") has been proposed as a scenario for a first galaxy (Frebel \& Bromm 2012), while the fourth scenario ("Two Bursts") is similar to the Brown et al. (2014) history for Ursa Major I. The fifth panel ("Two Bursts 75/25") shows two bursts with the first containing $75 \%$ of the stars, similar to the Brown et al. (2014) star formation histories for Hercules and Leo IV.

The lower three panels of Figure 1 show data from observations of Segue 1 (Frebel et al. 2014) and Reticulum 2 (Simon et al. 2015) as well as the combined data from the six UFDs observed by Brown et al. (2014) along with Segue 2 (Kirby et al. 2013), Segue 1, and Reticulum 2. While Reticulum 2 could be explained by a number of possible scenarios, Segue 1 is highly unusual. The closest match would be a three-burst scenario with smaller dispersion than shown in the "Three Bursts" panel. The overall UFD population shares qualitative features with the continuous Type II + Ia model, but this could also be caused by it being a composite of a large number of bursts across many systems.

\subsection{Clustering in Segue 1}

Figure 2 shows how unusual Segue 1 is compared to the overall UFD population. For all UFDs except Segue 1, the majority of stars have metallicities within 0.5 dex of the mean. In Segue 1, five of the seven stars have metallicities outside the range of $>90 \%$ of the stars in UFDs. As well as the extreme metallicities, any scenario to explain the star formation history of Segue 1 needs to explain the gaps in metallicity between the three stars with $[\mathrm{Fe} / \mathrm{H}] \approx-3.6$, the two with $[\mathrm{Fe} / \mathrm{H}] \approx-2.4$, and the two with $[\mathrm{Fe} / \mathrm{H}] \approx-1.5$. No such gap is seen anywhere else in MDFs of UFDs with the exception of Canes Venatici II, which contains a single high-metallicity star. The favored star formation history for Canes Venatici II from Brown et al. (2014) is two bursts with the second burst separated from the first by 3.2 Gyr containing only $5 \%$ of the stars, so this higher-metallicity star is likely part of a small second burst.

As a statistical test to compare a three-cluster model to an unclustered model, we use the Beale (1969) pseudo- $F$ statistic:

$$
F^{*}=\frac{J_{1}^{2}-J_{2}^{2}}{J_{2}^{2}} \frac{\left(N-c_{2}\right) c_{2}^{-2 / p}}{(N-1)-\left(N-c_{2}\right) c_{2}^{-2 / p}}
$$




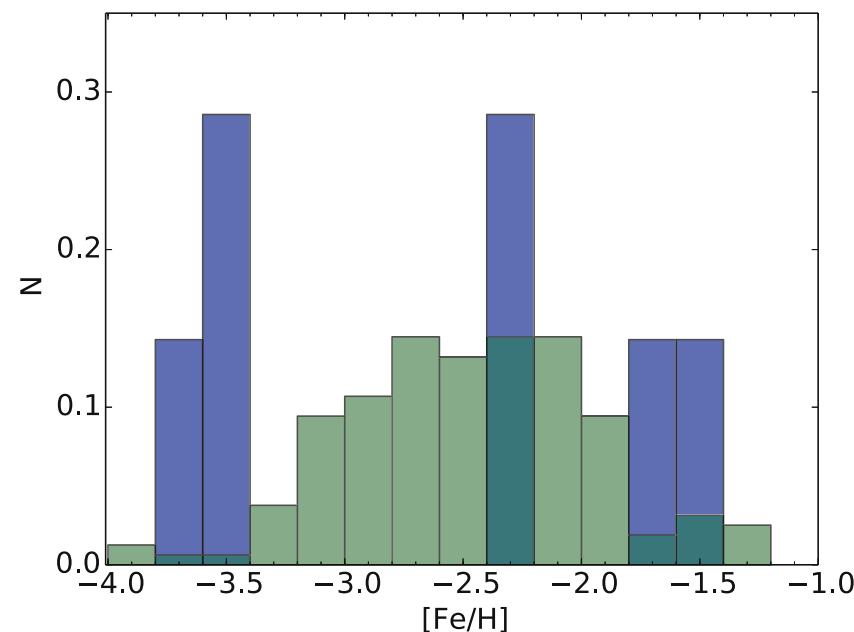

Figure 2. Normalized MDF of Segue 1 (Frebel et al. 2014) (in blue) and the combined normalized MDF of Bootes I, Coma Berenices, Canes Venatici II, Hercules, and Leo IV and (in green) Ursa Major I (Brown et al. 2014), Reticulum 2 (Simon et al. 2015), and Segue 2 (Kirby et al. 2013).

where $J_{1}^{2}=\Sigma_{i}\left(x_{i}-\mu\right)^{2}$ and $J_{2}^{2}=\Sigma_{k} \Sigma_{k_{i}}\left(x_{k_{i}}-\mu_{k}\right)^{2}$ correspond to the squared errors of the unclustered and clustered models, $\mu_{k}$ are the cluster centroids, $N$ is the number of data points, $c_{2}$ is the number of clusters, and $p$ is the dimensionality of the data. The first term is a comparison of how well the data matches the models while the second term includes only the number of clusters, number of observations, and the dimensionality of the data such that models with more parameters are penalized.

For a three-cluster model with centroids at $(-3.65,-2.36,-1.55)$ compared to a model without clusters, $F^{*}=274 \approx F(2,4)_{p=0.0001}$, showing that the three-cluster model is a better fit to the data. The three-cluster model also outperforms the best two-cluster model with $F^{*}=7 \approx$ $F(1,4)_{p=0.05}$.

Gaps in the MDF can be produced by star formation in discrete bursts. Single-age bursts should produce groups of stars that form at about the same time and therefore have similar metallicities. However, even three bursts centered on the metallicity of the three groups in Segue 1 will not necessarily produce an MDF like in Segue 1. While there may be gaps in the MDF, typically one or more of the groups will contain either no stars or only one star, which does not conclusively show that the groups exist. Put differently, it is possible that there are systems with a similar star formation history to Segue 1 where we will not observe one or more of the groups and the system will therefore not be recognized as having grouped star formation.

There are good physical and observational reasons to expect that star formation should be clustered in time and/or place in low-mass systems. In the local universe stars are observed to form in clusters (Lada \& Lada 2003). If this is also the case at the very low gas masses and star formation rates of the UFDs as suggested by Clark et al. (2008), we would expect to observe groups of stars with the same chemical composition. In larger systems this effect is washed out because eventually stars exist at all metallicities. However, in a "first galaxy" such as Segue 1, there are no later generations, meaning that the signature of the first clusters is observed. As shown in BlandHawthorn et al. (2015) and Webster et al. (2014), in a dark

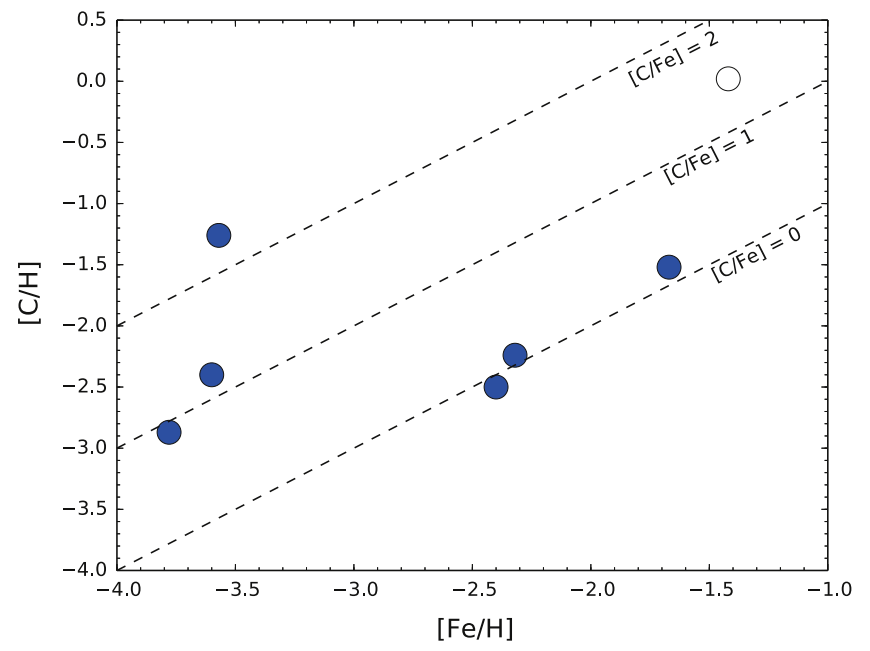

Figure 3. $[\mathrm{C} / \mathrm{H}]$ vs. $[\mathrm{Fe} / \mathrm{H}]$ for Segue 1 , with dashed lines denoting lines of constant $[\mathrm{C} / \mathrm{Fe}]$. The empty circle indicates the star which has been carbonenhanced by its binary companion, while the filled-in circles are stars that likely retained the abundance of the gas in which they formed. The data is from Frebel et al. (2014) and Norris et al. (2010b).

matter halo that is small enough, a single supernova can affect the entire system with star formation only possible during sufficiently long breaks between supernovae. If there is a large number of supernovae during a short period of time, the gas may be blown out completely and permanently end star formation in the system.

The unusual MDF of Segue 1 is likely connected to its status as a first galaxy, with both features caused by its inability to retain gas for more than $\sim 50 \mathrm{Myr}$. This is plausible if Segue 1 had a mass of $M_{\text {vir }} \sim 10^{7} M_{\odot}$ when it formed stars at $z>10$, which would imply a current mass of $M_{\mathrm{vir}}=10^{8-9} M_{\odot}$ (Webster et al. 2015). For Segue 1 the observed half-light mass of $M_{\text {half }}=2 \pm 1 \times 10^{5} M_{\odot}$ and half-light radius of $r_{\text {half }} \approx 30 \mathrm{pc}$ (Collins et al. 2014) give a best-fit mass assuming an NFW profile of $M_{\mathrm{vir}} \sim 3 \times 10^{9} M_{\odot}$, but with an uncertainty of more than an order of magnitude.

Given that continuous enrichment is unlikely, Segue 1 must have formed its stars in separate bursts. However, these bursts cannot have been similar to those determined by Brown et al. (2014), which produced stars with a range of $[\mathrm{Fe} / \mathrm{H}]$ and $[\alpha / \mathrm{Fe}]$ and likely had some self-enrichment (Webster et al. 2015). For Segue 1 the three bursts each contain red giants that are consistent within the uncertainties with the stars within each cluster having the same $[\mathrm{Fe} / \mathrm{H}]$.

\subsection{Carbon}

In the Milky Way halo and in UFDs, a large proportion of very metal-poor stars are enhanced in carbon relative to iron. This is seen in Segue 1 where four of the seven stars with measured abundances are carbon-enhanced metal-poor (CEMP) stars with $[\mathrm{C} / \mathrm{Fe}]>0.7$, although one of the four appears to have received carbon from a binary companion (Frebel et al. 2014). This star is excluded from the discussion below. The scatter $\Delta[\mathrm{C} / \mathrm{Fe}]$ is $>2$ dex, is significantly larger than for heavier elements in Segue 1, suggesting that carbon enrichment was decoupled from the enrichment of heavier elements (see also Frebel et al. 2014; Ritter et al. 2015; Sluder et al. 2015). 
Figure 3 shows the relationship between $[\mathrm{C} / \mathrm{H}]$ and $[\mathrm{Fe} / \mathrm{H}]$ along with lines representing constant $[\mathrm{C} / \mathrm{Fe}]$. There is no clear trend of $[\mathrm{C} / \mathrm{H}]$ with $[\mathrm{Fe} / \mathrm{H}]$. This is suggestive that the stars with higher $[\mathrm{Fe} / \mathrm{H}]$ in Segue 1 need not be a consequence of a large number of enrichment events but are instead the result of inhomogeneous mixing. With few events, the inherent scatter that results from a single supernova becomes more important as we will see in Section 3.

Figure 3 also provides some additional evidence for clustered star formation in Segue 1 in that despite the high overall scatter in carbon abundances, the two stars with $[\mathrm{Fe} / \mathrm{H}] \approx-2.4$ have similar $[\mathrm{C} / \mathrm{H}]$, as do two of the three stars with $[\mathrm{Fe} / \mathrm{H}] \approx-3.5$. The only outlier as classified by the $\mathrm{Fe}-$ clusters is the star with $[\mathrm{C} / \mathrm{H}]=-1.25$ at $[\mathrm{Fe} / \mathrm{H}]=-3.57$. This star is from a different observational sample than the others and is also an outlier in $[\mathrm{Mg} / \mathrm{Fe}],[\mathrm{Al} / \mathrm{Fe}]$, and $[\mathrm{Ni} / \mathrm{Fe}]$ (Norris et al. 2010b; Frebel et al. 2014).

Ritter et al. (2015) explain stars with enhanced $[\mathrm{C} / \mathrm{Fe}]$ as resulting from differences in the entropy of the inner and outer regions of the supernova ejecta. In their simulations the gas that first returns to the center is deficient in the innermost $10 \%$ of the ejecta, which contains iron peak and $\alpha$ elements. The outer ejecta cools first and does not rise to the same radii as the inner ejecta. The outer ejecta collapses to the center of the system resulting in carbon-enhanced, iron-poor star formation. In Segue 1 there is low variance in $[\mathrm{X} / \mathrm{Fe}]$ abundances of stars for $\alpha$ and $\mathrm{Fe}-$ peak elements, but $[\mathrm{C} / \mathrm{Fe}]$ does vary significantly between clusters. This is consistent with the Ritter et al. (2015) single and clustered supernovae simulations.

\section{SCENARIOS FOR THE STAR FORMATION HISTORY OF SEGUE 1}

In the discussion above we considered only the gaps in the MDF and were not concerned with the precise metallicities. We have not yet provided a solution as to how stars with metallicities as high as $[\mathrm{Fe} / \mathrm{H}] \sim-1.5$ can form. No other UFD contains more than one observed star with $[\mathrm{Fe} / \mathrm{H}]$ more than 1 dex higher than its mean $[\mathrm{Fe} / \mathrm{H}]$. This is despite the fact that unlike Segue 1, most of these systems likely had a longer star formation history as they show evidence of enrichment from AGB stars and SNe Ia.

Reaching an average $[\mathrm{Fe} / \mathrm{H}] \approx-1.5$ with only $\mathrm{SNe}$ II from the $[\mathrm{Fe} / \mathrm{H}] \approx-3.6$ in the lowest metallicity, three stars would require enrichment by $\approx 70$ events per $10^{5} M_{\odot}$ of gas. The stellar mass of $1500 M_{\odot}$ in Segue 1 given a Kroupa (2001) IMF therefore implies too few enrichment events. A top-heavy IMF (Geha et al. 2013) could cause sufficient enrichment events, but it is likely that 70 supernovae per $10^{5} M_{\odot}$ of gas within $50 \mathrm{Myr}$ would blow out most of the metals and much of the gas (Mac Low \& Ferrara 1999; Webster et al. 2014).

Therefore, inhomogeneous mixing must be at least part of the explanation for the highest metallicity stars. However, gas with a metallicity of $[\mathrm{Fe} / \mathrm{H}]=-1.5$ rarely shows densities sufficient for star formation in gas accreted onto a dark matter halo (Greif et al. 2010) or after a single supernova event (Bland-Hawthorn et al. 2015). Any proposed star formation history must not only explain why there are no stars with metallicities between $[\mathrm{Fe} / \mathrm{H}]=-1.7$ and -2.3 or between $[\mathrm{Fe} / \mathrm{H}]=-2.5$ and -3.4 , but also how stars with such a high metallicity can form despite the short timescale of star formation in the system.
We consider three possibilities to explain the MDF. In the first, the clusters of higher-metallicity stars form at the interface of two colliding supernova remnants where dense gas is swept up and partially mixed with the ejecta. The second scenario has the entire system enriched to $[\mathrm{Fe} / \mathrm{H}] \approx-2.5$ by $\sim 10 \mathrm{SNe}$, with no star formation possible in between because each supernova temporarily pushes out the dense gas. The $[\mathrm{Fe} / \mathrm{H}] \approx-1.5$ stars form from the first dense gas that returns to the center in a pause between supernovae, with the $[\mathrm{Fe} / \mathrm{H}] \approx-2.5$ stars forming shortly after. Finally, we consider the possibility of enrichment by exotic types of $\mathrm{SNe}$ such as pair-instability supernovae.

\subsection{Simulations}

Our hydrodynamical simulations are described in detail in Bland-Hawthorn et al. (2015). We used the Fyris Alpha hydrodynamical code (Sutherland 2010), which solves ideal Euler hydrodynamical flows of gas with cooling and an equation of state appropriate to astrophysical gases in a fixed gravitational potential. A nested three-level grid was set up with each level having $216^{3}$ cells and being a factor of 3 smaller than its outer containing level. The largest level covers the halo out to beyond the virial radius of $630 \mathrm{pc}$ while the level containing the inner region has a resolution of $1.4 \mathrm{pc}$ per cell. At $t=0$, a $25 M_{\odot}$ star is assumed to form in a low-mass $\left(M_{\mathrm{vir}}=10^{6}-10^{7} M_{\odot}\right)$ dark matter halo.

The effect on the gas, which was initially set up as a fractal ISM in approximate equilibrium, was traced for $25 \mathrm{Myr}$ with a resolution of $0.5 \mathrm{Myr}$. The models include the energy output of a $25 M_{\odot}$ star both during the main sequence lifetime and from the supernova after $6 \mathrm{Myr}$. The ionization phase from the precursor star was modeled using the MAPPINGS IV ionization code (Allen et al. 2008) for the thermal and ionization structures, the ATLAS9 atmospheric grid (Castelli \& Kurucz 2004), and the Meynet \& Maeder (2002) evolutionary tracks. The full details are in Section 2 of Webster et al. (2014). The supernova was modeled by inserting a bubble of hot gas with an equivalent energy of $10^{51} \mathrm{erg}$. As the highpressure bubble expands, its thermal energy is converted to kinetic energy and radiative losses. We assume the star explodes as a typical core-collapse supernova. Faint supernovae (e.g., Cooke \& Madau 2014) and other exotic supernovae will be discussed in Section 3.4.

The ionization and supernova from a $25 M_{\odot}$ star permanently switches off star formation for systems with $M \lesssim$ $10^{6.5} M_{\odot}$, but above this limit dark matter halos retain sufficient baryons to continue forming stars. A star positioned away from the center couples less efficiently with the dense gas with much of the energy escaping in the directions away from the center. This results in more gas retention and less enrichment. In a $10^{7} M_{\odot}$ dark matter halo with a baryon fraction of $10 \%$, star formation is switched off for $\sim 10 \mathrm{Myr}$ after the explosion of a high-mass star near the center but continues almost unaffected if the progenitor star is $\gtrsim 80 \mathrm{pc}$ away from the center (Webster et al. 2014).

In this section we consider two variants of the above model as approximations to two possible star formation histories of Segue 1. The first involves the collision of a centered and offcentered explosion, while the second models a single supernova after the gas has been enriched to $[\mathrm{Fe} / \mathrm{H}]=-2.5$. 


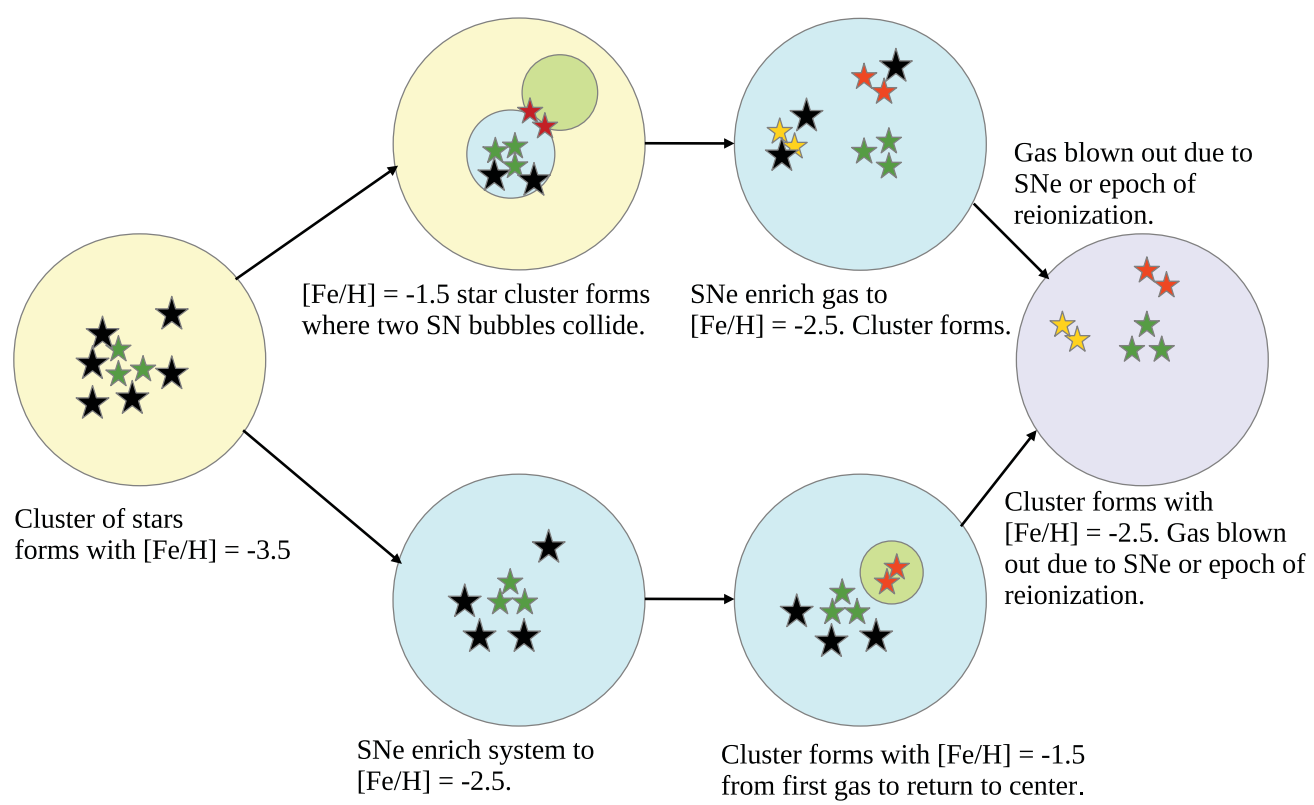

Figure 4. Two possible star formation histories for Segue 1. Both models start with a cluster or clusters of stars forming at $[\mathrm{Fe} / \mathrm{H}] \approx-3.5$ (green stars) and finish with stars with the metallicities observed in Segue 1 today. The upper track has a cluster of stars forming at $[\mathrm{Fe} / \mathrm{H}] \approx-1.5$ (red stars) at the collision of two supernova remnants. Subsequent supernovae enrich the gas to $[\mathrm{Fe} / \mathrm{H}] \approx-2.5$, at which time another cluster of stars forms (yellow stars). Star formation is then terminated within $50 \mathrm{Myr}$ of the first cluster forming. In the lower track the gas is first enriched to $[\mathrm{Fe} / \mathrm{H}]-2.5$ by $\sim 10$ supernovae, with little star formation between them. There is then a gap between supernovae in which gas returns to the center. The first gas to return is more metal-rich and forms a cluster of stars with $[\mathrm{Fe} / \mathrm{H}] \approx-1.5$ (red stars) and the cluster at $[\mathrm{Fe} / \mathrm{H}] \approx-2.5$ (yellow stars) forming later when the gas is more well-mixed. Star formation is then terminated either by supernovae or a global event such as reionization.

\subsection{Merging Supernova Remnants}

One possible scenario for the star formation history of Segue 1 involves the highest metallicity stars forming at the site where two supernova remnants collide. A single supernova expanding in an $\mathrm{H}_{\text {II }}$ region can also induce star formation (Whalen et al. 2008; Nagakura et al. 2009), but here we investigate the colliding case, which allows for higher metallicities to be reached. This possibility was briefly considered in relation to first galaxies by Greif et al. (2007), who noted that if a collision between SN remnants occurred early in their evolution in an overdense region the density could become high enough for gravitational fragmentation and therefore star formation.

As predicted by Ostriker \& McKee (1988), a supernova remnant develops a dense shell of gas when its age is approximately equal to the cooling time of the shock-heated gas. Numerical simulations of the ISM of the Milky Way (Rosen \& Bregman 1995; Korpi et al. 1999; de Avillez 2000; Joung \& Mac Low 2006) have found that when these shells collide, dense and cold filamentary clouds are formed due to thermal instabilities and supersonic turbulence, an effect first noted for the case of two interacting supernova remnants by Bodenheimer et al. (1984). In our simulations, given gas densities of $\rho>1 \mathrm{~cm}^{-3}$, which are observed in the swept-up gas, the cooling time is shorter than our 500 kyr time resolution and star formation is therefore possible. If sufficient metal mixing occurs in the swept-up gas, the stars will form at a high metallicity. To investigate this scenario we used the Fyris Alpha simulations discussed briefly in Section 3.1 and in detail in Bland-Hawthorn et al. (2015).

The scenario is illustrated in the upper path of Figure 4. At $t=0$, there is a dark matter halo containing gas enriched to $[\mathrm{Fe} / \mathrm{H}]=-4$. This starting metallicity is uncertain and could be as high as $[\mathrm{Fe} / \mathrm{H}]=-3.5$. In this gas a cluster of stars forms. This cluster includes stars with masses of $M \approx 0.75 M_{\odot}$, observed as red giants today (green stars in Figure 4) along with at least one star with $M>8 M_{\odot}$ (black stars in Figure 4). At a similar time, a cluster forms in lower density gas well away from the center with at least one massive star. The two clusters contain the three stars with metallicities of approximately $[\mathrm{Fe} / \mathrm{H}]=-3.5$, observed as red giants today.

One supernova then occurs in each of the clusters described above. The inner supernova ejects $0.08 M_{\odot}$ of $\mathrm{Fe}$ into a region with $2 \times 10^{4} M_{\odot}$ of gas enriching it to $\overline{[\mathrm{Fe} / \mathrm{H}]}=-2.5$. At the same time the supernova further out, which occurs in lessdense gas, has enriched a region with $2 \times 10^{3} M_{\odot}$ of gas to $\overline{[\mathrm{Fe} / \mathrm{H}]}=-1.5$. These supernova remnants expand, sweep up gas, and collide, inducing star formation in the swept-up gas. A cluster of stars forms with $[\mathrm{Fe} / \mathrm{H}]=-1.5$ (red stars in Figure 4). The density and metallicity in the simulations at the time when the two supernovae remnants collide is shown in Figure 5. Note that these simulations are unable to describe the complex physics of colliding supernova remnants, so here we are interested only in establishing whether the scenario is plausible.

Subsequent supernovae then enrich the gas to $[\mathrm{Fe} / \mathrm{H}]=$ -2.5 and a cluster forms at $[\mathrm{Fe} / \mathrm{H}] \approx-2.5$ containing two low-mass stars (yellow in Figure 4) that will be red giants today. These stars must form less than $50 \mathrm{Myr}$ after the first cluster of stars because stars in Segue 1 do not show evidence of AGB or Type Ia enrichment. Finally, gas is blown out, terminating star formation. The gas can be blown out either by multiple closely spaced supernovae or as a result of the epoch of reionization. As noted by Nichols \& Bland-Hawthorn (2011), heated gas can be removed through tidal stripping by the host galaxy or ram pressure stripping by a tenuous a hot halo. 

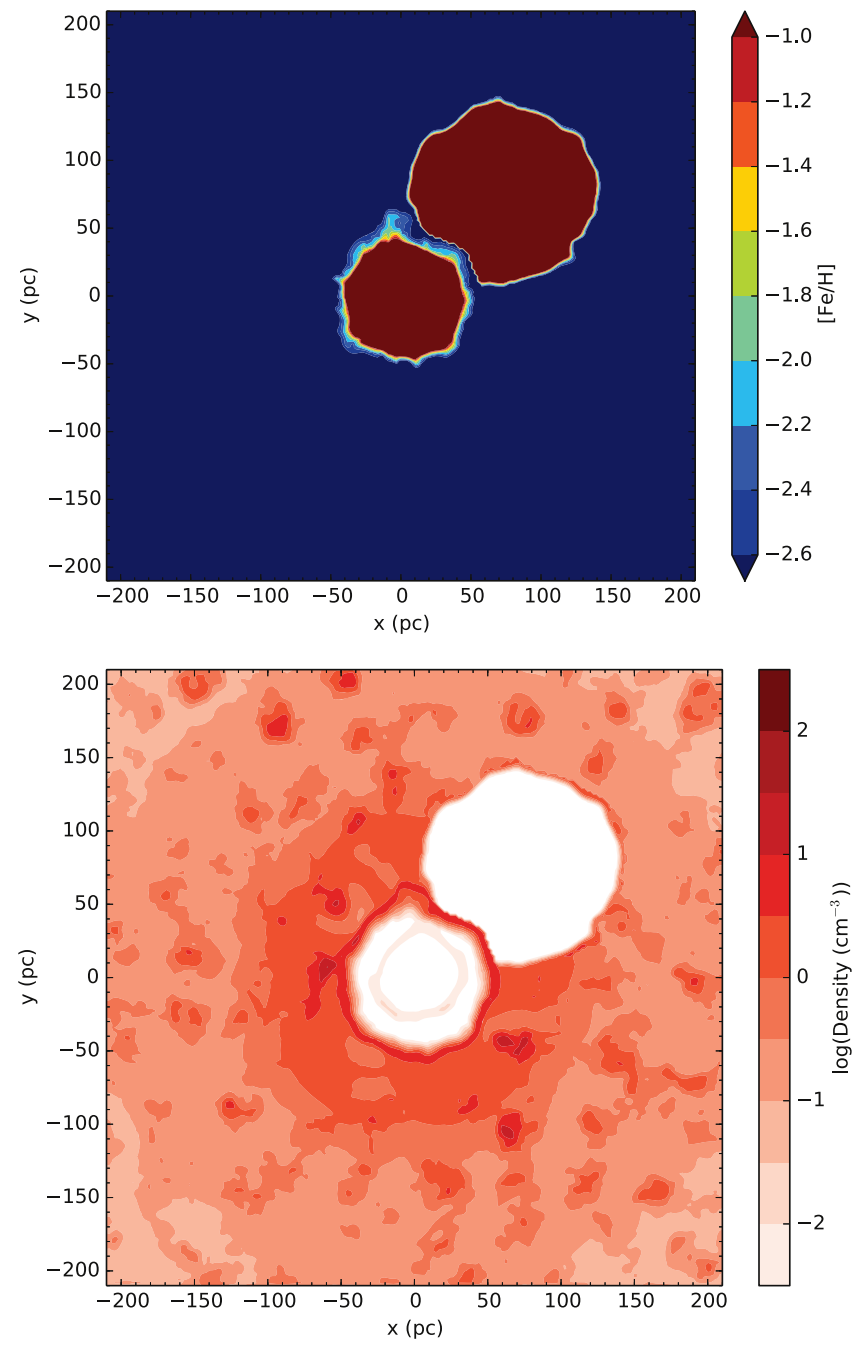

Figure 5. A central slice of the metallicity and density of the gas just before the collision of supernova remnants from central and off-centered explosions. The two remnants have swept-up dense neutral gas.

In the merging supernova remnants scenario, the reason that stars form at $[\mathrm{Fe} / \mathrm{H}] \approx-1.5$ is that this is the metallicity of dense gas swept up and mixed with the supernova ejecta. In the future we will seek to perform hydrodynamical simulations of the effects of colliding supernova remnants, including whether the conditions for star formation exist, but this is beyond the scope of the present work. Here we simply note that at the boundary of the remnants, gas with $[\mathrm{Fe} / \mathrm{H}]$ similar to the highest metallicity stars can build up, such that given the right conditions stars will form. The stars at $[\mathrm{Fe} / \mathrm{H}] \approx-2.4$ may be formed slightly later at another site along the boundary between the two remnants or by the first dense gas that returns to the center of the system after $\sim 10 \mathrm{Myr}$, which is more metal-rich than the mean metallicity as we will see in Section 3.3.

We therefore suggest the following explanation for the MDF in Segue 1. The first stars formed with $[\mathrm{Fe} / \mathrm{H}] \approx-3.6$, near the mean metallicity of the system when it formed. The first two SNe occurred at approximately the same time, separated by at least $\sim 75 \mathrm{pc}$. Dense gas was swept up and partially mixed with the supernova ejecta. A shock formed and the gas cooled, resulting in a cluster of stars forming with $[\mathrm{Fe} / \mathrm{H}]$ much higher than the mean metallicity of the system. All the star formation in the system could be complete within 10-15 Myr, before supernova feedback blew all the gas out. A top-heavy initial mass function as suggested by Geha et al. (2013) is expected to produce 250-400 supernovae in Segue 1 (Frebel et al. 2014), which could easily blow the gas out of an $M_{\mathrm{vir}} \sim 10^{7} M_{\odot}$ dark matter halo.

This scenario is particularly interesting in light of the Ritter et al. (2015) simulations discussed in Section 2.4. They found enhanced carbon relative to iron for gas which falls into the center. The iron-rich ejecta has more entropy and reaches higher radii than the carbon-rich ejecta, resulting in the stars which form at the center being carbon-enhanced. Here we instead have stars forming at higher radii where two supernova remnants merge. If the iron has overtaken the carbon by the time of the collision, the stars which form will have high $[\mathrm{Fe} / \mathrm{H}]$ and low $[\mathrm{C} / \mathrm{Fe}]$. This is observed in the star at $[\mathrm{Fe} / \mathrm{H}]=-1.5$ with $[\mathrm{C} / \mathrm{Fe}] \sim 0$. It should be noted that Ritter et al. (2015) model lower-mass $\left(10^{6} M_{\odot}\right)$ systems over longer time periods, but it is suggestive of a possibility for inhomogeneous enrichment.

\subsection{Two Bursts}

Another possible star formation history for Segue 1 is shown in the lower track of Figure 4 and can be summarized as follows:

1. A cluster of second-generation stars formed at $t=0$ with $[\mathrm{Fe} / \mathrm{H}] \approx-3.5$ (shown in green in Figure 4).

2. Within the $30-50 \mathrm{Myr}$ allowed for star formation, $\approx 10$ $\mathrm{SNe}$ resulted from the star formation of this first cluster, enriching the medium to $[\mathrm{Fe} / \mathrm{H}] \approx-2.5$. This supernova rate is too high for the gas to recover between events so little or no star formation occurs during this time.

3. Due to the stochasticity of supernova events at low star formation rates, there was a sufficiently long gap $T \sim 10 \mathrm{Myr}$ between supernovae, allowing the gas to return to the center. The first gas to reach the center can have metallicities as high as $[\mathrm{Fe} / \mathrm{H}] \approx-1.5$ and reach sufficient densities that stars may form (red stars in Figure 4). Gas in different parts of the system remained at $[\mathrm{Fe} / \mathrm{H}] \approx-2.5$ and formed another cluster (yellow stars in Figure 4).

4. As a result of the star formation from these clusters, or due to the epoch of reionization or other environmental effects, the star-forming gas was evaporated or blown out of the system, resulting in the termination of star formation.

We investigated this possibility using the Fyris Alpha simulations described in Section 3.1 and in Bland-Hawthorn et al. (2015). However instead of a uniform starting metallicity of $[\mathrm{Fe} / \mathrm{H}]=-4$, we assumed a starting metallicity of $[\mathrm{Fe} / \mathrm{H}]=-2.5$, such that the simulation models only the final supernova in step 2 of the scenario discussed above. In the initial state of the simulation we assume that all the gas has this starting metallicity. In reality it is likely that the gas will not be this well-mixed and that regions of higher metallicity will exist. These pre-existing inhomogeneities may assist in reaching metallicities higher than we find.

We assume that a supernova occurs in a medium already somewhat disrupted by the previous supernovae. While BlandHawthorn et al. (2015) models a $25 M_{\odot}$ star, here the star will be lower-mass because $\sim 10$ supernovae from the cluster have already occurred, suggesting a timescale of 20-30 Myr. This 

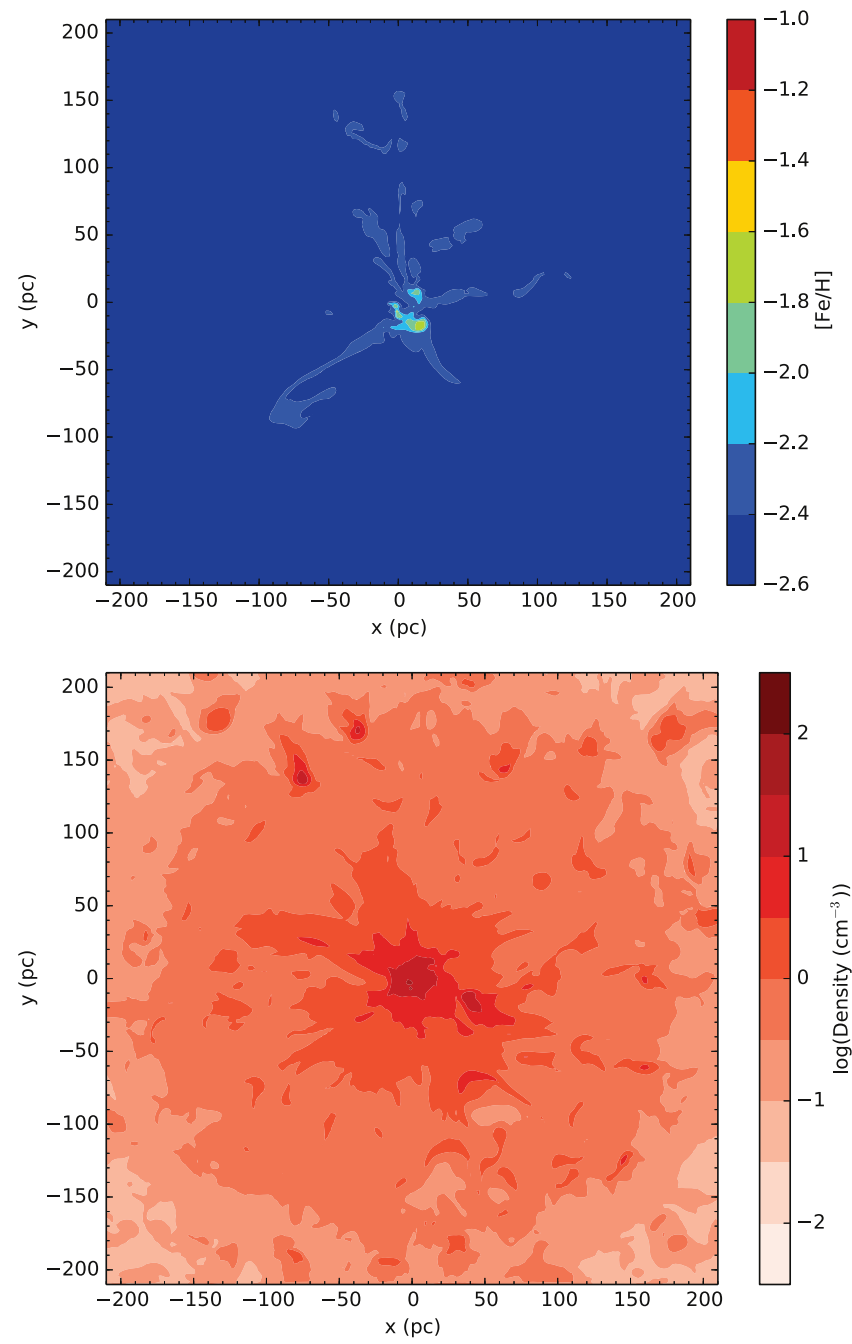

Figure 6. A central slice of the metallicity and density of the gas $11 \mathrm{Myr}$ after a supernova when the first dense gas is returning to the center.

implies a mass of $<15 M_{\odot}$. However, the effect of the previous supernovae will be such that the surrounding medium is likely to be largely evacuated. As an approximation, the supernova is therefore assumed to occur in the same medium as that for a $25 M_{\odot}$ star.

The effect of the supernova is shown in Figure 6. The gas that returns to the center after $\sim 10 \mathrm{Myr}$ is enriched to higher $[\mathrm{Fe} / \mathrm{H}]$ then the overall average. While the metallicity of the gas in the central $20 \mathrm{pc}$ has a mean of $[\mathrm{Fe} / \mathrm{H}] \approx-2.0$, there are pockets of dense gas with metallicities of $[\mathrm{Fe} / \mathrm{H}] \gtrsim-1.6$. For example, $11 \mathrm{Myr}$ after the supernova, there is a clump of gas with a mass of $200 M_{\odot}$ and $[\mathrm{Fe} / \mathrm{H}]=-1.6$ within an approximately spherical region with a radius of $7 \mathrm{pc}$.

The third cluster observed today may form at a similar time in one of the lower metallicity clumps. The combined effect of the supernovae from these two newly formed clusters or the onset of the epoch of reionization may then eject all the gas in the system such that the three clusters described above contain all the stars that ever formed in Segue 1.

This scenario provides a natural explanation of the observed MDF. The first cluster at $[\mathrm{Fe} / \mathrm{H}] \approx-3.5$ is responsible for the three lowest metallicity stars observed as red giants today. The supernovae resulting from this cluster enrich the ISM to $[\mathrm{Fe} / \mathrm{H}] \approx-2.5$ while blowing the gas out of the center such that stars with metallicities in the range of $-3.5<[\mathrm{Fe} / \mathrm{H}]$ $<-2.5$ are prevented from forming. The other two groups of stars form during a gap between supernovae before star formation is permanently switched off.

The abundances of elements relative to iron should then be close to the average SN II yields, although there may be a bias toward the higher mass stars, which explode first. The first 10 supernovae may not be the entire supernova output of the first cluster, in which case the lower-mass supernova progenitors would not yet have exploded by the time the higher-metallicity clusters in Segue 1 form. This is indeed seen with $[\alpha / \mathrm{Fe}] \sim 0.5$.

Under this scenario part of the explanation for Segue 1 is that it has just the right mass and supernova rate that star formation can be temporarily switched off by supernova feedback without the gas being blown away completely. Such a scenario may be rare which is why we have seen it only once in the UFDs discovered so far.

\subsection{Exotic Supernovae}

A possible explanation for high $[\mathrm{Fe} / \mathrm{H}]$ in a first galaxy is a pair-instability supernova (PISN). A single PISN in a precursor minihalo could enrich a halo with $10^{5} M_{\odot}$ to an average of $[\mathrm{Fe} / \mathrm{H}] \approx-2.5$. (Heger et al. 2003) There may then be sufficient natural scatter to explain the existence of stars at $[\mathrm{Fe} / \mathrm{H}] \approx-3.5$ and -1.5 . The suppressed $[\mathrm{Ba} / \mathrm{Fe}]$ and $[\mathrm{Sr} / \mathrm{Fe}]$ in Segue 1 also support this explanation. However, measurements of $\alpha$ abundances as well as $[\mathrm{Co} / \mathrm{Fe}]$ (Norris et al. 2010a; Frebel et al. 2014) do not match the distinctive signatures expected for a PISN (Heger \& Woosley 2002). The suggestion that different elements mix differently into the ISM (Ritter et al. 2015; Sluder et al. 2015) may be a partial solution to this; however, this effect does not explain why all the stars in Segue 1 show similar abundance patterns for all $\alpha$ and Fe-peak elements.

Other alternatives to regular core-collapse supernovae do not match the abundance patterns of Segue 1. Pulsational pairinstability supernovae eject mostly lighter elements, with the iron lost as the star ends its life as a black hole (Heger et al. 2003), so are not a solution to the observed high $[\mathrm{Fe} / \mathrm{H}]$. Hypernovae can eject more iron into the ISM than regular supernovae, but only by a factor of 2-3 (Nomoto et al. 2006). They are more energetic than regular supernova by an order of magnitude, so will blow out a large proportion of gas and metals from a low-mass system. Faint supernovae, which produce small amounts of iron compared to carbon, have been suggested by Cooke \& Madau (2014) as an explanation for the formation of CEMP stars in minihalos, as their lower energy means they are less efficient at blowing out gas. However, they eject only a small amount of iron and would therefore struggle to explain the higher-metallicity stars in Segue 1 .

It is therefore unlikely that exotic types of supernovae are responsible for the observed abundances of Segue 1. However, a pair-instability supernova could produce a similar $[\mathrm{Fe} / \mathrm{H}]$ distribution, so it should be considered if other systems showing similar MDFs to Segue 1 are discovered.

\section{DISCUSSION}

\subsection{The Nature and Enrichment History of Segue 1}

Segue 1 provides a unique opportunity to investigate the early metal buildup in galaxies because it experienced so few 
chemical enrichment events. In all other known galaxies, enrichment from later generations complicates the picture. UFDs were likely some of the early building blocks of the Milky Way halo, so by studying galaxies like Segue 1 we can help determine the chemical history of the Milky Way.

Our results point to a new way of looking at metal buildup. When there are few previous enrichment events, abundances at a given location can be dominated by the effect of the last supernova. $[\mathrm{Fe} / \mathrm{H}]$ and $[\mathrm{C} / \mathrm{H}]$ do not increase uniformly everywhere in a galaxy, but are instead highly inhomogeneous with some places reaching high metallicity before further mixing takes them back to close to the average metallicity for the galaxy. This "two steps forward, one step back" enrichment means that some relatively metal-rich stars form before lowermetallicity stars.

Therefore, while after many supernovae there is a gradual increase in average metallicity over time, this is not necessarily the case locally and over shorter periods of time. Hence, the oldest stars are not necessarily the most metal-poor stars and, more importantly, ancient metal-rich stars are expected to exist. None of the scenarios that we consider plausible for Segue 1 has the lowest metallicity stars enriching the gas that forms stars with moderate metallicity, which then enriches the gas that forms the highest metallicity stars. We find that the most likely scenarios have either the lowest metallicity of $[\mathrm{Fe} / \mathrm{H}] \approx-3.6$ stars enrich the gas that forms both the higher-metallicity clusters, which share the same enrichment history, or that all stars in Segue 1 were formed from gas with the same enrichment history. Furthermore, given that they require metallicities much higher than the likely average of the gas in Segue 1 , the $[\mathrm{Fe} / \mathrm{H}] \approx-1.5$ stars are more likely to have formed before the $[\mathrm{Fe} / \mathrm{H}] \approx-2.4$ stars. Gaia will provide accurate ages for very metal-poor halo stars and can test for such out of order enrichment. If our view of Segue 1 and the chemical enrichment of early systems is correct, we predict that some of the oldest stars may have higher metallicities of $[\mathrm{Fe} / \mathrm{H}]>-2.0$.

In systems with few enrichment events, short-term variations in the environment can result in a large variance in $[\mathrm{Fe} / \mathrm{H}]$. The first stars in the system can therefore be outliers in chemical abundance space. However if star formation is clustered, groups of stars form with very similar abundances. The early outliers in abundance space resulting from the varied environment come in groups of stars rather than individually. This means that rather than each individual system having a small number of outlier stars, some systems may have a large number (if they contain a cluster or clusters which is an outlier in abundance space) and others might have none (if the early clusters are not outliers). In this way, we explain how five of the seven stars in Segue 1 can have $\Delta[\mathrm{Fe} / \mathrm{H}]>0.8$ dex from the mean, whereas most other UFDs have much narrower MDFs. The MDFs seen in the majority of UFDs can result either from the system having a large number of enrichment events, filling in the MDF and hiding distinct clusters, or by only having a few clusters with similar $[\mathrm{Fe} / \mathrm{H}]$. The latter class of systems do not form outlier stars at any time.

For most UFDs the overall picture is one of early environment-dependent enrichment, followed by increasing homogeneity after sufficient enrichment events have occurred. In this way we can account for both overall homogeneity in the abundances of most stars (Cayrel et al. 2004) along with the unusual abundances of others (Frebel \& Norris 2015; Hansen et al. 2015). The unusual stars, which are not necessarily the most metal-poor, are those which formed first.

Our work is in line with that of Ji et al. (2015), who suggested that CEMP stars are second-generation stars enriched only by massive Pop III stars. Subsequent generations quickly wash out this carbon enhancement. This fits with Segue 1 , where the stars with $[\mathrm{Fe} / \mathrm{H}] \approx-3.5$ are CEMP stars, while the higher-metallicity stars, which in our models are formed from gas enriched by these second-generation stars, all have $[\mathrm{C} / \mathrm{Fe}] \approx 0$. Furthermore, it is another example of outlier stars being the first to form. The yields of Population III stars appear to be determined best by studying stars in dwarf galaxies and the Milky Way halo which have unusual abundances.

The discovery of 20 new UFDs in 2015 is likely to help answer the question of whether Segue 1 is a rare system or if its chemical abundances are common for the faintest galaxies. Its MDF is unusual compared to all other known UFDs, but nearly all of them are more luminous systems with longer star formation histories.

\subsection{Reticulum 2}

The UFD Reticulum 2 is similar to Segue 1 in its distance, mean metallicity, and luminosity. The system was discovered only recently, so at the time of writing only iron (Koposov et al. 2015b; Simon et al. 2015; Walker et al. 2015) and mediumresolution $(R \sim 18,000) \alpha$ abundances (Koposov et al. 2015b) have been determined. As shown in Figure 1 its MDF appears similar to the more luminous UFDs, contrary to Segue 1. However this does not rule out a similar star formation history to Segue 1.

In our scenarios above, the $[\mathrm{Fe} / \mathrm{H}]=-2.4$ stars in Segue 1 formed from gas enriched by the first cluster of stars at $[\mathrm{Fe} / \mathrm{H}]=-3.5$. This produced a gap in the MDF, but equally plausible scenarios may not form such a gap. Because of the logarithmic nature of $[\mathrm{Fe} / \mathrm{H}]$, a first cluster at $[\mathrm{Fe} / \mathrm{H}]=-3.0$ would require $>75 \%$ of the number of supernovae to reach $[\mathrm{Fe} / \mathrm{H}]=-2.5$ as a first cluster at $[\mathrm{Fe} / \mathrm{H}]=-3.5$. It would be much more difficult to distinguish between two clusters with means separated by $0.5 \mathrm{dex}$ than between two clusters separated by 1 dex.

The scenarios in Section 3 may not always lead to the formation of stars with $[\mathrm{Fe} / \mathrm{H}] \approx-1.5$ will form. Star formation in merging supernova remnants may not be common or the gas which returns to the center first may not form stars (or as in Ritter et al. (2015) this gas may be carbon-rich and iron-poor). It is therefore possible that we will find many more examples of Ret 2-like MDFs than Segue 1 even if both are first galaxies and similar processes occur in both systems. The new galaxies discovered by DES provide an excellent opportunity to test this.

There are multiple possible explanations for the origin of Ret 2. The first is that it has a similar history to Segue 1 with clusters of stars forming at $[\mathrm{Fe} / \mathrm{H}] \approx-3.2$ and $[\mathrm{Fe} / \mathrm{H}] \approx-2.5$, but no higher-metallicity cluster (or a small high-metallicity cluster from which we have not yet observed stars). Alternatively, the MDF of Ret 2 is consistent with a gradual buildup of metallicities as in the "Type II" model described in Section 2.1, or at the other extreme, a single burst with $\overline{[\mathrm{Fe} / \mathrm{H}]} \approx-2.7$ and $\sigma \approx 0.3$. In Segue 1 we are fortunate that the clusters are well-separated, allowing us to distinguish between these models. 
Ret 2 is the closest of these new systems at $30 \mathrm{kpc}$ (Koposov et al. 2015a) and is therefore the best candidate for performing high-resolution abundance measurements. High-resolution spectroscopy is required to determine whether Ret 2 passes the tests for a "first galaxy" as outlined in Frebel \& Bromm (2012). A first galaxy should not show stars even at $[\mathrm{Fe} / \mathrm{H}]$ $>-2$, with $[\alpha / \mathrm{Fe}]$ systematically lower than the halo value of 0.35 . Furthermore, there should be no signature of s-process or carbon enrichment from AGB stars. Medium-resolution observations of Ret 2 (Koposov et al. 2015b) provide a tentative suggestion that $[\alpha / \mathrm{Fe}]$ does not decline with increased $[\mathrm{Fe} / \mathrm{H}]$, with all 17 observed stars showing $[\alpha / \mathrm{Fe}]>0.2$, close to the mean $[\alpha / \mathrm{Fe}]=0.4$. High-resolution observations are required to test this more rigorously as well as to determine the presence or absence of neutron-capture elements.

Segue 1 is highly unusual compared with all other observed galaxies. However, first galaxies may be difficult to observe because they are likely to be intrinsically less luminous due to their shorter period of star formation, so this could be a result of an observational bias. If Ret 2 proves to be similar to Segue 1, there will be two known first galaxies helping us to determine which features are typical of first galaxies and which are unique to individual systems.

\section{SUMMARY}

We have used the MDF of Segue 1 to reconstruct its star formation history and to learn about the nature of first and early galaxies. In Section 2 we showed that Segue 1 is best explained by clustered star formation. A gradual increase in $[\mathrm{Fe} / \mathrm{H}]$ with time cannot reproduce its MDF. There are three distinct groups in $[\mathrm{Fe} / \mathrm{H}]$ with gaps of 1.2 and $0.55 \mathrm{dex}$. All other UFDs observed to date have $90 \%$ of their stars within 0.5 dex of the mean $[\mathrm{Fe} / \mathrm{H}]$ for the system and few show stars with metallicities as low as $[\mathrm{Fe} / \mathrm{H}] \approx-3.6$ or as high as $[\mathrm{Fe} / \mathrm{H}] \approx-1.5$ as in Segue 1 . We conclude that the unusual MDF is a sign of earliest star formation in line with Segue 1 being a "first galaxy," meaning that it experienced only a single burst of star formation lasting $\lesssim 50 \mathrm{Myr}$. There are a number of features of Segue 1 that are naturally explained by the first galaxy hypothesis:

1. The gaps in the MDF are caused by the stochasticity within a single generation of star formation. Segue 1 is the only known galaxy that appears not to have experienced self-enrichment and therefore multiple generations of star formation. Multiple generations of star formation are likely to wash out stochastic effects, resulting in the MDF being filled in near the average metallicity.

2. The highest metallicity stars remain difficult to explain, but may be related to the large variation in metallicity caused by even a single supernova in a low-mass system. First galaxies are more likely to have low masses because they are more susceptible to effects such as supernova feedback and the epoch of reionization, which can quench their star formation.

3. Segue 1 has the lowest intrinsic luminosity of any known galaxy. A Kroupa IMF implies that it has only formed only $\approx 1500 M_{\odot}$ of stars over its lifetime. This low amount of total star formation is likely related to the short duration of star formation.
In Section 3, we used hydrodynamical simulations to test two possible explanations for the MDF of Segue 1. One possibility is that the high-metallicity stars formed from sweptup gas at the interface between two colliding supernova remnants. In this scenario clusters of stars with a difference in $[\mathrm{Fe} / \mathrm{H}]$ of 2 dex can form within $10 \mathrm{Myr}$ of each other. Furthermore, as shown by Ritter et al. (2015) and discussed in Section 2.4 , there can be large variance in $[\mathrm{C} / \mathrm{Fe}]$ at early times after a supernova explosion in a low-mass system. In particular, the iron-rich ejecta has a higher entropy and can overtake the carbon-rich ejecta. If this occurs by the time of the collision it provides an explanation for $[\mathrm{C} / \mathrm{Fe}]$ being suppressed by $1-2$ dex for the stars at $[\mathrm{Fe} / \mathrm{H}] \approx-1.5$ compared with those at $[\mathrm{Fe} / \mathrm{H}] \approx-3.5$.

Alternatively, our hydrodynamic simulations show that in an $M_{\mathrm{vir}}=10^{7} M_{\odot}$ dark matter halo with $10^{6} M_{\odot}$ of gas preenriched to $[\mathrm{Fe} / \mathrm{H}]=-2.5$, the first gas that returns to the center of the system after a supernova explosion will be enriched to $[\mathrm{Fe} / \mathrm{H}] \approx-1.5$ and be sufficiently dense that star formation is plausible. We also briefly explored the possibility of more exotic types of supernovae such as PISNs to reproduce the MDF of Segue 1, but these could not explain the abundances of other elements.

At the time of writing, 17 new UFD candidates have been detected in DES (Drlica-Wagner et al. 2015; Koposov et al. 2015a). One of these, Reticulum 2, which has been confirmed as a galaxy, is at a similar distance as Segue 1 and has a similar mean metallicity. Medium-resolution observations of $[\alpha / \mathrm{Fe}]$ suggest it as a tentative candidate for a first galaxy. Highresolution abundance measurements are required to determine whether this is indeed the case. Segue 1 is the only known first galaxy and it is therefore currently impossible to say whether its features are typical or unusual. The newly discovered systems provide an exciting opportunity to test this. In the longterm, LSST will be capable of detecting much fainter objects while G-CLEF on the GMT will enable high-resolution abundance measurements of fainter stars.

D.W. is supported by an ARC Laureate Fellowship awarded to JBH. We acknowledge the Dick Hunstead Fund for Astrophysics for funding A.F.'s visit to the University of Sydney to work on this paper. A.F. acknowledges support from the Silverman (1968) Family Career Development Professorship. A.F. is supported by NSF grant AST-1255160. This research was also supported in part by the National Science Foundation under Grant No. NSF PHY11-25915, as the collaboration was formed at the Kavli Institute at UC Santa Barbara during the Galactic Archaeology and Precision Stellar Astrophysics program. We thank the referee for insightful comments that led to significant improvements to this work.

\section{REFERENCES}

Allen, M. G., Groves, B. A., Dopita, M. A., Sutherland, R. S., \& Kewley, L. J. 2008, ApJS, 178, 20

Asplund, M., Grevesse, N., \& Sauval, A. J. 2005, in ASP Conf. Ser. 336, Cosmic Abundances as Records of Stellar Evolution and Nucleosynthesis, ed. T. G. Barnes, III, \& F. N. Bash (San Francisco, CA: ASP), 25

Beale, E. 1969, Euclidean Cluster Analysis (Milton Keynes: Scientific Control Systems Limited)

Belokurov, V., Zucker, D. B., Evans, N. W., et al. 2007, ApJ, 654, 897

Bigiel, F., Leroy, A., Walter, F., et al. 2008, AJ, 136, 2846

Bland-Hawthorn, J., Karlsson, T., Sharma, S., Krumholz, M., \& Silk, J. 2010a, ApJ, 721, 582

Bland-Hawthorn, J., Krumholz, M. R., \& Freeman, K. 2010b, ApJ, 713, 166 
Bland-Hawthorn, J., Sutherland, R., \& Webster, D. 2015, ApJ, 807, 154

Bodenheimer, P., Yorke, H. W., \& Tenorio-Tagle, G. 1984, A\&A, 138, 215

Bovill, M. S., \& Ricotti, M. 2009, ApJ, 693, 1859

Bromm, V., \& Yoshida, N. 2011, ARA\&A, 49, 373

Brown, T. M., Tumlinson, J., Geha, M., et al. 2012, ApJL, 753, L21

Brown, T. M., Tumlinson, J., Geha, M., et al. 2014, ApJ, 796, 91

Castelli, F., \& Kurucz, R. L. 2004, arXiv:astro-ph/0405087

Cayrel, R., Depagne, E., Spite, M., et al. 2004, A\&A, 416, 1117

Clark, P. C., Glover, S. C. O., \& Klessen, R. S. 2008, ApJ, 672, 757

Collins, M. L. M., Chapman, S. C., Rich, R. M., et al. 2014, ApJ, 783, 7

Cooke, R. J., \& Madau, P. 2014, ApJ, 791, 116

de Avillez, M. A. 2000, MNRAS, 315, 479

De Silva, G. M., Freeman, K. C., Bland-Hawthorn, J., Asplund, M., \& Bessell, M. S. 2007, AJ, 133, 694

De Silva, G. M., Sneden, C., Paulson, D. B., et al. 2006, AJ, 131, 455

Drlica-Wagner, A., Bechtol, K., Rykoff, E. S., et al. 2015, ApJ, 813, 109

Feng, Y., \& Krumholz, M. R. 2014, Natur, 513, 523

Frebel, A., \& Bromm, V. 2012, ApJ, 759, 115

Frebel, A., \& Norris, J. E. 2015, ARA\&A, 53, 631

Frebel, A., Simon, J. D., \& Kirby, E. N. 2014, ApJ, 786, 74

Geha, M., Brown, T. M., Tumlinson, J., et al. 2013, ApJ, 771, 29

Geha, M., Willman, B., Simon, J. D., et al. 2009, ApJ, 692, 1464

Greif, T. H., Glover, S. C. O., Bromm, V., \& Klessen, R. S. 2010, ApJ, 716,510

Greif, T. H., Johnson, J. L., Bromm, V., \& Klessen, R. S. 2007, ApJ, 670,1

Hansen, T., Hansen, C. J., Christlieb, N., et al. 2015, ApJ, 807, 173

Heger, A., \& Woosley, S. E. 2002, ApJ, 567, 532

Heger, A., Woosley, S. E., Langanke, K., et al. 2003, NuPhA, 718, 159

Iwamoto, K., Brachwitz, F., Nomoto, K., et al. 1999, ApJS, 125, 439

Ji, A. P., Frebel, A., \& Bromm, V. 2015, MNRAS, 454, 659

Joung, M. K. R., \& Mac Low, M.-M. 2006, ApJ, 653, 1266

Karlsson, T., Bland-Hawthorn, J., Freeman, K. C., \& Silk, J. 2012, ApJ, 759,111
Karlsson, T., Bromm, V., \& Bland-Hawthorn, J. 2013, RvMP, 85, 809

Kirby, E. N., Boylan-Kolchin, M., Cohen, J. G., et al. 2013, ApJ, 770, 16

Koposov, S. E., Belokurov, V., Torrealba, G., \& Evans, N. W. 2015a, ApJ, 805, 130

Koposov, S. E., Casey, A. R., Belokurov, V., et al. 2015b, ApJ, 811, 62

Korpi, M. J., Brandenburg, A., Shukurov, A., Tuominen, I., \& Nordlund, A. 1999, ApJL, 514, L99

Kroupa, P. 2001, MNRAS, 322, 231

Lada, C. J., \& Lada, E. A. 2003, ARA\&A, 41, 57

Larsen, S. S., Strader, J., \& Brodie, J. P. 2012, A\&A, 544, L14

Mac Low, M.-M., \& Ferrara, A. 1999, ApJ, 513, 142

Meynet, G., \& Maeder, A. 2002, A\&A, 390, 561

Nagakura, T., Hosokawa, T., \& Omukai, K. 2009, MNRAS, 399, 2183

Nichols, M., \& Bland-Hawthorn, J. 2011, ApJ, 732, 17

Nomoto, K., Tominaga, N., Umeda, H., Kobayashi, C., \& Maeda, K. 2006, $\mathrm{NuPhA}, 777,424$

Norris, J. E., Gilmore, G., Wyse, R. F. G., Yong, D., \& Frebel, A. 2010a, ApJL, 722, L104

Norris, J. E., Wyse, R. F. G., Gilmore, G., et al. 2010b, ApJ, 723, 1632

Ostriker, J. P., \& McKee, C. F. 1988, RvMP, 60, 1

Ricotti, M., \& Gnedin, N. Y. 2005, ApJ, 629, 259

Ritter, J. S., Sluder, A., Safranek-Shrader, C., Milosavljević, M., \& Bromm, V. 2015, MNRAS, 451, 1190

Rosen, A., \& Bregman, J. N. 1995, ApJ, 440, 634

Simon, J. D., Drlica-Wagner, A., Li, T. S., et al. 2015, ApJ, 808, 95

Sluder, A., Ritter, J., Safranek-Shrader, C., Milosavljevic, M., \& Bromm, V. 2015, arXiv:1505.07126

Sutherland, R. S. 2010, Astrophysics Source Code Library, record, ascl: 1010.015

Walker, M. G., Mateo, M., Olszewski, E. W., et al. 2015, ApJ, 808, 108

Webster, D., Bland-Hawthorn, J., \& Sutherland, R. 2015, ApJL, 799, L21

Webster, D., Sutherland, R., \& Bland-Hawthorn, J. 2014, ApJ, 796, 11

Whalen, D., van Veelen, B., O'Shea, B. W., \& Norman, M. L. 2008, ApJ, 682,49 